\title{
PARALLELIZABLE MANIFOLDS AND THE FUNDAMENTAL GROUP
}

\author{
F. E. A. JOHNSON AND J. P. WALTON
}

\$0. Introduction. Low-dimensional topology is dominated by the fundamental group. However, since every finitely presented group is the fundamental group of some closed 4-manifold, it is often stated that the effective influence of $\pi_{1}$ ends in dimension three. This is not quite true, however, and there are some interesting border disputes. In this paper, we show that, by imposing the extra condition of parallelizability on the tangent bundle, the dominion of $\pi_{1}$ is extended by an extra dimension. First, we prove

THEOREM A. For each $n \geqslant 5$, any finitely presented group may occur as the fundamental group of a smooth closed parallelizable n-manifold.

The proof may be compared with the usual proof that all finitely presented groups are realized as fundamental groups of closed 4-manifolds, but is nevertheless more delicate, because of the need to avoid the obstruction to parallelizability. In dimension 4 , the obstruction cannot be avoided. In fact, a smooth closed almost parallelizable manifold $M^{4}$ is parallelizable when both its signature $\operatorname{Sign}(M)$ and Euler characteristic $\chi(M)$ vanish. In particular, the vanishing of the Euler characteristic severely restricts the groups which can occur as fundamental groups of smooth closed parallelizable 4-manifolds. In fact, for many fundamental groups the Euler characteristic bounds the signature (see, for example, [4], [16]), but the relationship is neither universal nor straightforward.

To describe our results, it helps to partition the class of finitely presented groups as

$$
\Pi(4) \cup C(4),
$$

where $\Pi(4)$ is the class of fundamental groups of smooth closed parallelizable 4-manifolds, and $C(4)$ is the complementary class. An exact algebraic description of this partition seems very unlikely. Nevertheless it is possible to give some sufficient conditions for membership; the first involves membership of $\Pi(4)$.

THEOREM B. If $\Gamma$ admits a presentation of the form

$$
\Gamma=\left\langle X_{1}, \ldots, X_{g} ; W_{1}(X), \ldots, W_{r}(X)\right\rangle
$$

in which $r<g$, then $\Gamma \in \Pi(4)$. 
Our condition for membership of $C(4)$ is given in terms of low dimensional homological restrictions on a discrete group $\Gamma$; here $k$ is a field.

$(k): 2 \operatorname{dim}_{k} H^{1}(\Gamma, k) \leqslant 1+\operatorname{dim}_{k} H^{2}(\Gamma, k)$

, $(k): \operatorname{dim}_{k} H^{1}(\Gamma, k) \leqslant \operatorname{dim}_{k} H^{2}(\Gamma, k)$ and $H^{4}(\Gamma, k)=0$.

THEOREM C. C(4) contains all finitely presented groups which satisfy some condition $(k)$ where $k$ is a field, and all those which satisfy some condition o $(k)$ where $k$ is a field of characteristic other than 2.

We conclude by discussing the $\Pi(4)-C(4)$ partition with reference to some familiar infinite groups, and give some applications to combinatorial/geometric group theory.

The 4-dimensional results of this paper date from March 1991, and have circulated in a number of versions since then. Some thanks are long overdue, notably to Professor Hirzebruch and the staff of the Max-Planck Institut für Mathematik for their hospitality when the first-named author was visiting the Max-Planck Institut für Mathematik, Bonn in 1991, to Thomas Delzant, Dieter Kotschick, Ralph Strebel who saw the earliest version and made some useful comments, and to Hansjörg Geiges and Charles Thomas for comments on the material in Section 1.

$\S 1$. Framed surgery. We review briefly the theory of framed surgery on stably parallelizable manifolds. The standard reference for such matters is [9]. Throughout we shall assume that all manifolds are smooth, closed and connected; we denote by $M_{1} \# M_{2}$ the connected sum of manifolds $M_{1}, M_{2}$. A manifold $M^{n}$ is parallelizable when its tangent bundle $\tau_{M}$ is isomorphic, as a vector bundle, to the product bundle $\tau_{M} \cong M \times \mathbf{R}^{n} ; M^{n}$ is stably parallelizable when $\tau_{M}$ becomes trivial upon addition of a trivial line bundle $\tau_{M} \oplus \varepsilon \cong M \times \mathbf{R}^{n+1}$; finally, $M$ is almost parallelizable when $M-\{p t\}$ is parallelizable. We note the following, which is essentially a restatement of Lemma (3.4) of [9].

(1.1) Let $M$ be a smooth closed connected manifold. Then

$$
\begin{aligned}
M \text { is parallelizable } & \Rightarrow M \text { is stably parallelizable } \\
& \Rightarrow M \text { is almost parallelizable. }
\end{aligned}
$$

The following two propositions are straightforward.

(1.2) Let $M_{1}, M_{2}$ be closed smooth manifolds. If $M_{1}$ is stably parallelizable and $M_{2}$ is parallelizable, then $M_{1} \times M_{2}$ is parallelizable.

(1.3) Let $M_{1}, M_{2}$ be closed smooth stably parallelizable manifolds. Then $M_{1} \# M_{2}$ is also stably parallelizable. 

Put

Now let $\Gamma=\left\langle X_{1}, \ldots, X_{g} ; W_{1}(X), \ldots, W_{r}(X)\right\rangle$ be a finitely presented group.

$$
M_{1}=\underbrace{\left(S^{1} \times S^{n-1}\right) \#\left(S^{1} \times S^{n-1}\right) \# \cdots \#\left(S^{1} \times S^{n-1}\right)}_{g} .
$$

Then $\pi_{1}\left(M_{1}\right)$ is a free group of rank $g$. We may identify the canonical generators of $\pi_{1}\left(M_{1}\right)$ with $X_{1}, \ldots, X_{g}$. Observe that $M_{1}$ is stably parallelizable, since $S^{1} \times S^{n-1}$ is parallelizable. We may represent each relator $W_{j}(X)$ by a closed path $\lambda_{j}: S^{\prime} \rightarrow M_{1}$. By a standard general position argument, we may assume that $\lambda_{1}, \ldots, \lambda_{r}$ are all imbeddings, with empty mutual intersections. Since $M_{1}$ is stably parallelizable, the normal bundle to any $\lambda_{j}$ is trivial. Let $M$ be obtained from $M_{1}$ by framed surgery on $\lambda_{1}, \ldots, \lambda_{r}$. Then we obtain the classical result

(1.4) Let $\Gamma=\left\langle X_{1}, \ldots, X_{g} ; W_{1}(X), \ldots, W_{r}(X)\right\rangle$ be a finitely presented group. Then, for each $n \geqslant 4$, there exists a smooth closed stably parallelizable $n$-manifold $M^{n}$ such that $\pi_{1}\left(M^{n}\right) \cong \Gamma$.

If $M^{n}$ is a closed almost parallelizable $n$-manifold, then the obstruction to parallelizability is a single element

$$
\psi(M) \in H^{n}\left(M ; \pi_{n-1}(S O(n))\right) \cong \pi_{n-1}(S O(n)) .
$$

Let $\sigma: \pi_{n-1}(S O(n)) \rightarrow \pi_{n-1}(S O)$ denote the stabilization map, and put

$$
\not{ }_{n}=\operatorname{Ker}\left(\sigma: \pi_{n-1}(S O(n)) \rightarrow \pi_{n-1}(S O) .\right.
$$

Then $\sigma(\psi(M))$ represents the obstruction to the almost parallelizable manifold $M$ being stably parallelizable, so that, when $M$ is stably parallelizable, $\psi(M) \epsilon$ $H^{n}\left(M ; \mathscr{H}_{n}\right) \cong \mathscr{H}_{n}$. We can rephrase matters as follows.

(1.5) Let $M^{n}$ be a smooth closed connected stably parallelizable $n$-manifold. Then there is a single obstruction $\psi(M) \in H^{n}\left(M ; \mathscr{H}_{n}\right) \cong{ }_{n}$ which vanishes precisely when $M$ is parallelizable.

The groups $/$ have been computed by Kervaire [8], using earlier work of Paechter [14]. When $n$ is even, $\mathbb{Z}_{n} \cong \mathbb{Z}$, and, in this case, the obstruction $\psi(M)$ to parallelizability of the stably parallelizable manifold $M^{n}$ may be identified with the Euler characteristic $\chi(M)$. When $n$ is odd, $\mathscr{T}_{n}$ is isomorphic to $\mathbb{Z} / 2 \mathbb{Z}$, except in three special dimensions $n=1,3,7$, where one has $y_{1} \cong y_{3} \cong \pi_{7} \cong 0$; in these dimensions, stably parallelizable manifolds are necessarily parallelizable.

Proof of Theorem A. The first three cases, $n=5,6,7$, are essentially different, and we give separate arguments for each. By means of (1.4), we may first construct a smooth closed stably parallelizable $n$-manifold $M_{1}^{n}$ such that $\pi_{1}\left(M_{1}\right) \cong \Gamma$. Then $M_{1}$ is orientable and so satisfies Poincaré Duality with rational coefficients. 
If $n=5$, the obstruction to parallelizability $\psi(M)$ can be identified with the mod 2 semi-characteristic of $M$, namely

$$
\psi(M)=\sum_{r=0}^{2} \operatorname{dim}_{\mathbb{F}_{2}} H_{r}\left(M ; \mathbb{F}_{2}\right)(\bmod 2),
$$

where $\mathbb{F}_{2}$ is the field with two elements. This follows easily from (9.2) of [7], or rather from the equivalent statement in cohomology.

Thus, if $\psi\left(M_{1}\right) \neq 0$, then $\psi\left(M_{1} \#\left(S^{2} \times S^{3}\right)\right)=0$. Hence either $M_{1}$ or $M_{1} \#\left(S^{2} \times S^{3}\right)$ is parallelizable, so that the desired result follows, since $\pi_{1}\left(M_{1} \#\left(S^{2} \times S^{3}\right)\right) \cong \pi_{1}\left(M_{1}\right) \cong \Gamma$.

If $n=6$, then the Euler characteristic of $M_{1}$ is even. In fact, we have

$$
\chi\left(M_{1}\right)=2\left(1-\beta_{1}+\beta_{2}\right)-\beta_{3},
$$

where $\beta_{k}$ is the $k$ th (rational) Betti number. However, the middle dimensional intersection form is here skew-symmetric and non-degenerate, so that $\beta_{3}$ is also even. Thus we may write $\chi\left(M_{1}\right)=2 k$. If $k=0$, put $M=M_{1}$. Then $\psi(M)=$ $\chi\left(M_{1}\right)=0$, and so $M$ is parallelizable. If $k>0$, put

$$
M=M_{1} \#(\underbrace{\left.S^{3} \times S^{3}\right) \# \cdots \#\left(S^{3} \times S^{3}\right)}_{k},
$$

whilst if $k>0$, put

$$
M=M_{1} \#(\underbrace{\left.S^{2} \times S^{4}\right) \# \cdots \#\left(S^{2} \times S^{4}\right)}_{k},
$$

Either way, $M$ is stably parallelizable with $\pi_{1}(M) \cong \pi_{1}\left(M_{1}\right) \cong \Gamma$. However $\psi(M)=\chi(M)=0$, so that $M$ is parallelizable as desired.

If $n=7$, there is nothing left to do, since $\mathscr{K}_{7}=0$ and $M_{1}$ is automatically parallelizable.

If $n>7$, then we may write $n=m+3 p$, where $m=5,6,7$. By (1.3), we can find a smooth closed stably parallelizable $m$-manifold $X^{m}$ with $\pi_{1}(X) \cong \Gamma$. Put

$$
M^{n}=X^{m} \times \underbrace{S^{3} \times S^{3} \times \cdots \times S^{3}}_{p} .
$$

Then $M$ is parallelizable by (1.2), and $\pi_{1}(M) \cong \Gamma$. This completes the proof.

§. Proofs of Theorems B and C. The above discussion shows that, for stably parallelizable 4-manifolds, the Euler characteristic may be identified with the obstruction to parallelizability of $M^{4}$. The problem of finding a smooth closed 4-manifold with given fundamental group and vanishing Euler characteristic was previously considered by Hausmann and Weinberger [2]; see also the article by Kotschick [10].

Proof of Theorem B. Let $\left\langle X_{1}, \ldots, X_{g} ; W_{1}(X), \ldots, W_{r}(X)\right\rangle$ be a finite presentation of the group $\Gamma$ for which $r<g$. Let $M_{0}$ be the smooth 4-manifold obtained by attaching $g$ 1-handles to $S^{4}$; then $\pi_{1}\left(M_{0}\right)$ is a free group $F_{g}$ of rank $g$, and the cores of the attached 1-handles may be identified with a set of free generators $X_{1}, \ldots, X_{g}$. Moreover,

$$
\chi\left(M_{0}\right)=-2(g-1) .
$$


Using general position, we may represent $W_{1}(X), \ldots, W_{r}(X)$ by a collection of $r$ imbedded frame 1-spheres $\Sigma_{1}, \ldots, \Sigma_{r}$. Let $M_{1}$ be formed by framed surgery on $\Sigma_{1}, \ldots, \Sigma_{r}$. Then $M_{1}$ is a smooth closed stably parallelizable 4-manifold with $\pi_{1}\left(M_{1}\right)=\Gamma$, and

$$
\chi\left(M_{1}\right)=-2(g-r-1) .
$$

Observe that $\chi\left(M_{1}\right) \leqslant 0$, since $(r+1) \leqslant g$. If $r+1=g$, we are finished on putting $M=M_{1}$, since $\chi\left(M_{1}\right)=0$. If $r+1<g$, put

$$
M=\underbrace{M_{1} \#\left(S^{2} \times S^{2}\right) \#, \ldots, \#\left(S^{2} \times S^{2}\right)}_{g-r-1} .
$$

Then again $\chi(M)=0$, and $M$ is a closed parallelizable 4-manifold with $\pi_{1}(M)=\Gamma$. In either case, it follows that $\Gamma \in \Pi(4)$.

Proof of Theorem C. Let $M$ be a smoth closed orientable 4-manifold for which $\chi(M)=0$, and let $c: M \rightarrow K(\Gamma, 1)$ be a map inducing an isomorphism of fundamental groups. Write $h_{n}(\Gamma, k)=\operatorname{dim}_{k} H^{n}(\Gamma, k)$, where $k$ is a field. Observe that the induced map on cohomology $c^{*}: H^{i}(\Gamma, k) \rightarrow H^{i}(M, k)$ is an isomorphism for $i=1$, and is injective for $i=2$.

Writing $\operatorname{dim}_{k} H^{2}(M, k)=h_{2}(\Gamma, k)+e$ with $e \geqslant 0$, we have

$$
2-2 h_{1}(\Gamma, k)+h_{2}(\Gamma, k) \leqslant 2-2 h_{1}(\Gamma, k)+h_{2}(\Gamma, k)+e=\chi(M)=0,
$$

from which it follows that $2+\operatorname{dim}_{k} H^{2}(\Gamma, k) \leqslant 2 \operatorname{dim}_{k} H^{1}(\Gamma, k)$. Hence we see that, if $\Gamma$ belongs to $\Pi(4)$, then $\Gamma$ fails to satisfy conditions $\mathscr{L}(k)$ for any field $k$.

Suppose, in addition, that $k$ has characteristic different from 2, and that $\operatorname{dim}_{k} H^{1}(\Gamma, k) \leqslant \operatorname{dim}_{k} H^{2}(\Gamma, k)$ and $H^{4}(\Gamma, k)=0$. Let $x, y \in H^{2}(\Gamma, k)$; since $H^{4}(\Gamma, k)=0$ we see that $x \cup y=0 \in H^{4}(\Gamma, k)$. However, $c^{*}(x \cup y)=c^{*}(x) \cup$ $c^{*}(y)=0$, so that $c^{*}$ maps $H^{2}(\Gamma, k)$ isomorphically to a subspace of $H^{2}(M, k)$ which is totally isotropic with respect to the cup product form. Thus $2 h_{2}(\Gamma, k) \leqslant \operatorname{dim}_{k} H^{2}(M, k)$.

Writing $\operatorname{dim}_{k} H^{2}(M, k)=2 h_{2}(\Gamma, k)+e$ with $e \geqslant 0$, we see that

$$
\chi(M)=2-2 h_{1}(\Gamma, k)+2 h_{2}(\Gamma, k)+e=2+2\left(h_{2}-h_{1}\right)+e .
$$

Since $h_{1} \leqslant h_{2}$, we see that $\chi(M)>0$, which is a contradiction. Thus, when $\Gamma$ belongs to $\Pi(4), \Gamma$ fails to satisfy $h(k)$ for any field of characteristic other than 2 .

One should point out that the $/(k)$ conditions are related to an inequality which Epstein ([1]) attributes to Philip Hall.

\$3. The $\Pi(4)-C(4)$ partition. We begin with some general observations about the class $C(4)$. Let $\Gamma$ denote a finitely presented group. The abelianisation $\Gamma^{a b}$ may be identified with $H_{1}(\Gamma: \mathbb{Z})$, and when $\Gamma^{a b}$ is finite then $H^{1}(\Gamma \cdot \mathbb{Q}) \cong H_{1}(\Gamma: \mathbb{Q})=0$, so that $\Gamma$ satisfies the condition $(\mathbf{Q})$, that is,

(3.1) If $\Gamma$ is finitely presented and $\Gamma^{a b}$ is finite, then $\Gamma \in C(4)$. 
It follows from the condition $h(k)$ that

(3.2) If $\Gamma$ satisfies (orientable) 3-dimensional Poincaré Duality over a field $k$ of characteristic other than 2 , then $\Gamma \in C(4)$.

Next consider products. It follows easily from the Künneth Theorem that

(3.3) If $\Gamma_{1}, \Gamma_{2}, \ldots, \Gamma_{n}$ all satisfy $H^{1}\left(\Gamma_{i}: \mathbb{Q}\right) \neq 0$, then $\Gamma_{1} \times \Gamma_{2} \times \cdots \times \Gamma_{n} \in C(4)$, provided that $n \geqslant 5$.

It follows from (3.1) and (3.3) that, for any finitely presented group $\Gamma$, the $n$-fold direct product $\Gamma^{(n)}$ belongs to $C(4)$ for all $n \geqslant 5$. It is tempting to conjecture that $C(4)$ is closed with respect to direct products.

Finally, observe that a finite covering of a parallelizable manifold is parallelizable. Thus $\Pi(4)$ is closed with respect to taking subgroups of finite index; dually

(3.4) C(4) is closed with respect to extension by finite groups.

We conclude by showing how some familiar groups are situated with respect to the $\Pi(4)-C(4)$ partition; we confine our attention to infinite groups since, by (3.1), all finite groups belong to $C(4)$.

The most familiar infinite groups are perhaps the free abelian groups $\mathbb{Z}^{n}$ with $n \geqslant 1$. By (3.2) and (3.3), these belong to $C(4)$, with $\mathbb{Z}, \mathbb{Z}^{2}, \mathbb{Z}^{4}$ as the only possible exceptions. In fact, these are genuine exceptions, being the fundamental groups of the parallelizable 4-manifolds $S^{1} \times S^{3}, S^{1} \times S^{1} \times S^{2}$ and $S^{1} \times S^{1} \times S^{1} \times S^{1}$, respectively.

By a direct argument on the connected sum of parallelizable 4-manifolds, after adding on a copy of $S^{2} \times S^{2}$ to kill the Euler characteristic, one may prove

(3.5) П(4) is closed under free products.

It follows that the non-abelian free groups $\mathbb{F}_{n}$, with $n \geqslant 2$, all belong to II(4). More generally

(3.6) П(4) contains all Fuchsian groups.

This follows from Theorem B, by the canonical description of Fuchsian groups as 1-relator groups [6].

Since the classical Wirtinger presentation of a knot group has deficiency equal to one, we see also that any knot group belong to $\Pi(4)$. The referee of a previous version of this article pointed out that, by Artin spinning, any knot group is the fundamental group of a ribbon 2-knot, so that there is a more general and geometrically natural observation that one can make, namely

(3.7) The fundamental group of any 2-knot complement belongs to $\Pi(4)$.

To see this, one does framed surgery on an embedded 2 -sphere in $S^{4}$, to obtain a stably parallelizable 4-manifold $M^{4}$ with $\chi(M)=0$. However, by Van 
Kampen's Theorem, $\pi_{1}(M) \cong \pi_{1}\left(S^{4} \backslash S^{2}\right)$; this line of argument seems to go back to Hausmann and Weinberger [2].

Next consider lattices in semisimple Lie groups. The simplest examples are lattices in $\mathrm{PSL}_{2}(\mathbb{R})$, that is, Fuchsian groups, and so belong to $\Pi(4)$ as already noted. By contrast, "most" semisimple lattices belong to $C(4)$. One may argue as follows. A lattice $\Gamma$ in a connected linear semisimple Lie group contains a subgroup $\Gamma_{0}$ of finite index which is a direct product

$$
\Gamma_{0} \cong \Gamma_{1} \times \cdots \times \Gamma_{n},
$$

where $\Gamma_{i}$ is an irreducible lattice in a linear semisimple Lie group $G_{i}$. If each $G_{i}$ has real rank at least 2, then, by a result of Kazhdan [5], Matsushima [13] and Wang [15], the abelianisation of each $\Gamma_{i}$ is finite; thus $\Gamma^{a b}$ is also finite, so that $\Gamma \in C(4)$ by (3.1).

The remaining cases, where some of the irreducible components have rank 1 , are more obscure. Even so, one can still say something; for example, if $\Gamma_{i}$ is Fuchsian for at least two indices $i$, then, after passing to subgroups of finite index for which $H_{1}\left(\Gamma_{i}: \mathbb{Q}\right)$ is suitably large, a Künneth argument shows that $\Gamma_{0}$ satisfies condition $(\mathbb{Q})$, hence $\Gamma \in C(4)$. Note also that a torsion free uniform lattice in $\mathrm{PSL}_{2}(\mathbb{C})$ satisfies 3-dimensional Poincaré Duality, and so belongs to $C(4)$.

By applying the $\cdot(k)$ conditions, we also obtain

(3.8) If $\Gamma$ is a finitely presented group satisfying 4-dimensional Poincaré Duality over a field $k$ of arbitrary characteristic, and if $\chi(\Gamma)>0$, then $\Gamma \in C(4)$.

From the examples given above, one might be forgiven for assuming that $\Pi(4)$ was a small and reasonably tractable class. This is far from the case, however; from Theorem $\mathrm{B}$, we obtain

(3.9) Let $\Gamma$ be a finitely presented group. Then $\Gamma * \mathbb{F}_{m} \in \Pi(4)$ for some large enough integer $m$.

In particular, every finitely presented group imbeds as a subgroup of an element of $\Pi(4)$. In fact, taking $\Gamma$ to be Higman's universal finitely presented group [3], and observing that $\Gamma$ is contained in $\Gamma * \mathbb{F}_{m}$, we see that

(3.10) There exists an element of $\Pi(4)$ which contains every other finitely presented group as a subgroup.

The above results have an application to combinatorial group theory which is worth pointing out. Say that a finitely presented group $\Gamma$ is overdetermined when every finite presentation for $\Gamma$ has at least as many relations as generators; then Theorem B implies 
In particular, we obtain

(3.12) Any irreducible lattice in a non-compact semisimple Lie group of rank at least 2 is overdetermined.

This does not seem to be well known, although we are informed that the question has recently been considered in the preprint by J. Lott [11]; the corresponding question for lattices in the rank 1 group $\operatorname{PSL}_{2}(\mathbb{C})$ has previously been considered, for example in [12].

Other classes of groups are also shown to be overdetermined by this method; for example, finite groups, $\mathrm{PD}^{3}$-groups, and $\mathrm{PD}^{4}$-groups $\Gamma$ with $\chi(\Gamma)>0$. The first two of these have more elementary proofs, but the result for $\mathrm{PD}^{4}$ groups seems to be not only new, but, since $\mathbb{Z}^{4} \in \Pi(4)$ and $\chi\left(\mathbb{Z}^{4}\right)=0$, also best possible; it is not known if there exists a $\operatorname{PD}^{4}$-group $\Gamma$ with $\chi(\Gamma)<0$.

\section{References}

1. D. B. A. Epstein. Finite presentations of groups and 3-manifolds. Quart. J. Math., 12 (1961), $205-212$.

2. J. C. Hausmann and S. Weinberger. Caractéristiques d'Euler et groupes fondamentaux des variétés de dimension 4. Comment. Math. Helv., 60 (1985), 139-144.

3. G. Higman. Subgroups of finitely presented groups. Proc. Roy. Soc. (Ser. A), 262 (1961), 455475.

4. F. E. A. Johnson and D. Kotschick. On the signature and Euler characteristic of certain fourmanifolds. Math. Proc. Camb. Phil. Soc., 114 (1993), 431-438.

5. D. A. Kahzdan. On the connection between the dual space of a group and the structure of its closed subgroups. Function. Anal. Appl., 1 (1967), 63-65.

6. S. Katok. Fuchsian Groups (University of Chicago Press, Chicago, 1992).

7. M. A. Kervaire. Relative characteristic classes. Amer. J. Math., 79 (1957), 517-558.

8. M. A. Kervaire. Some non-stable homotopy groups of Lie groups. Illinois J. Math., 4 (1960), $161-169$.

9. M. A. Kervaire and J. W. Milnor. Groups of homotopy spheres I. Ann. Math., 77 (1963), $504-537$

10. D. Kotshick. Four-manifold invariants of finitely presented groups. Preprint (University of Basle, 1993).

11. J. Lott. Deficiencies of lattice subgroups of Lie groups. Preprint (University of Michigan, 1997).

12. A. Lubotzky. Group presentation, $p$-adic analytic groups and lattices in $\mathrm{SL}_{2}(\mathrm{C})$. Ann. Math., 118 (1983), 115-130.

13. Y. Matsushima. On the first Betti number of compact quotient spaces of higher dimensional symmetric spaces. Ann. Math., 75 (1962), 312-330.

14. G. F. Paechter. The groups $\pi_{r}\left(V_{n, m}\right)$. Quart. J. Math. (Ser. 2), 7 (1956), 249-268.

15. S. P. Wang. The dual space of semisimple Lie groups. Amer. J. Math., 91 (1969), $921-937$.

16. H. E. Winkelnkemper, Un teorema sobre variedades de dimensión 4. Acta Mexicana Ci. Tecn., 2 (1968), 88-89.

Professor F. E. A. Johnson,

Department of Mathematics,

University College London,

Gower Street,

London, WC1E 6BT.

Dr. J. P. Walton,

Department of Mathematics,

University College London,

Gower Street,

London, WC1E 6BT.
57M50: MANIFOLDS AND CELL COMPLEXES: Low-dimensional topology; Geometric structures on low-dimensional manifolds. 